\title{
ANÁLISIS DE LA VISIBILIDAD INTERSITIO PARA EL PERÍODO TARDÍO E INCA DEL VALLE CALCHAQUÍ NORTE (SALTA, ARGENTINA)
}

\section{ANALYSIS OF INTERSITE VISIBILITY FOR THE LATE AND INCA PERIOD OF THE NORTHERN CALCHAQUÍ VALLEY (SALTA, ARGENTINA)}

\author{
María Cecilia Páez¹, Andrés Jakel², Luciano López ${ }^{3}$
}

${ }^{1}$ Consejo Nacional de Investigaciones Científicas y Técnicas. División Arqueología, Museo de Ciencias Naturales, Facultad de Ciencias Naturales y Museo. Universidad Nacional de La Plata, Paseo del Bosque s/n, B1900FWA, La Plata, Argentina Email: ceciliapaez@gmail.com ${ }^{2}$ Consejo Nacional de Investigaciones Científicas y Técnicas. División Arqueología, Museo de Ciencias Naturales, Facultad de Ciencias Naturales y Museo. Universidad Nacional de La Plata,

Paseo del Bosque s/n, B1900FWA, La Plata, Argentina. Email: andresjakel@gmail.com

${ }^{3}$ Consejo Nacional de Investigaciones Científicas y Técnicas. Instituto de Recursos Minerales, Facultad de Ciencias Naturales y Museo. Universidad Nacional de La Plata, Paseo del Bosque s/n, B1900FWA, La Plata, Argentina. Email: lopezluciano@hotmail.com

Presentado: $18 / 04 / 2020$

Aceptado: 30/03/2021

\section{Resumen}

La relación entre las lógicas organizativas locales e incaicas ha sido un constante foco de interés en el campo disciplinar a lo largo de toda el área andina. En sintonía, en el sector norte del Valle Calchaqui, hay un importante cúmulo de investigaciones que abordan la conformación de estos paisajes. Nuestro aporte en este sentido radica en analizar de qué manera la visibilidad entre los sitios con ocupación indígena local y aquellos que responden a una planificación estatal, habría condicionado la integración y articulación física y social del espacio calchaquí. Los resultados obtenidos sugieren, por un lado, que los sitios del Período Tardío habrían sido intervisibles, manifestando un manejo e integración del entorno mucho mayor que los sitios incaicos, para los que se presume una condición disruptiva. Por otro lado, pone en cuestión la idea de que los sitios con arquitectura incaica se localizarían en sectores con un mejor manejo visual del entorno.

Palabras clave: análisis del paisaje, SIG, Noroeste argentino, integración y articulación

\section{Abstract}

The relationship between local and Inca organizational logics has been a constant focus of interest in the disciplinary field throughout the Andean area. In the northern Calchaqui Valley there is an important body of research that addresses the conformation of these landscapes. Our contribution in this sense lies in analyzing how the visibility between sites with local indigenous occupation and those that respond to state planning, would have conditioned the integration and physical and social articulation of the Calchaqui space. The results obtained 
suggest, on the one hand, that the Late Period sites would have been intervisible, showing a much greater management and integration of the environment than the Inca sites, for which a disruptive condition is presumed. On the other hand, it questions the idea that sites with Inca architecture would be located in sectors with a better visual management of the environment.

Key words: landscape analysis, GIS, Northwest Argentina, integration and articulation

\section{Introducción}

El Valle Calchaquí atraviesa de $\mathrm{N}$ a S el sector centro-oeste de la provincia de Salta (NW de Argentina), franqueado por el río homónimo (Figura 1). Se divide en dos sectores de acuerdo a las diferencias climáticas y geomorfológicas. El sector norte se extiende hasta la quebrada de Angastaco y sierra de Apacheta, incluyendo los departamentos de La Poma, Cachi y Molinos (Zelarayán y Fernández 2015). La presente investigación estará situada en el departamento de Cachi, concretamente en el área de influencia de las localidades de Cachi y Payogasta.

La ocupación prehispánica del sector norte del Valle data de momentos tempranos, para los que se infiere un desarrollo aldeano con agricultura incipiente y recolección de productos vegetales (Rivolta y Cabral Ortiz 2017). Se trata de ocupaciones en terrazas bajas en las márgenes de los ríos, dentro de las cuales se destacan sitios como Campo Colorado, Salvatierra, Jaime, Cancha de Paleta, Tapia y Potrero Gutiérrez, entre otros (Díaz 1983; Tarragó y Díaz 1972, 1977; Tarragó y De Lorenzi 1976; Tarragó 1996; Rivolta et al. 2007; Rodríguez y Rivolta 2010; Seldes y Baffi 2016). El segundo milenio de la Era fue testigo de nuevas estrategias en el manejo del espacio, pautas de producción particulares, una organización jerárquica de la sociedad, con diferentes grados de control político a nivel regional, y relaciones asimétricas en la organización del trabajo, la distribución y el consumo de bienes (Tarragó 1995). Se registra un importante crecimiento demográfico, en coincidencia con el advenimiento de la agricultura extensiva acompañada por una tecnología de irrigación, así como diversificación y jerarquización de los asentamientos (DeMarrais 1997, Tarragó 1978). Destacan instalaciones como Ruiz de Los Llanos, Valdéz, RC-16, Quipón Arriba, Las Pailas, Corral del Algarrobal, Borgatta, Mariscal, El Tero, La Paya y Guitián, entre varios otros (Baldini y De Feo 2000; D’Altroy et al. 2000; DeMarrais 2001; Pollard 1983). En algunos de ellos, una serie de pequeñas estructuras de asentamiento tienden a agruparse a su alrededor, dando cuenta de la posible existencia de comunidades independientes hacia los últimos momentos del Período Tardío (DeMarrais 1997).

La presencia incaica se manifiesta en una arquitectura con rasgos cusqueños (Acuto y Gifford 2007; D'Altroy et al. 2000; DeMarrais 1997), en tanto continuaban desarrollándose las formas de organización política, tecnológica y social que caracterizaron los momentos previos. La manera en que se pusieron en relación ambas sociedades no muestra evidencias de fuerte conflictividad, al menos para este sector del Valle. Por el contrario y en sintonía con las propuestas para otras partes del área andina, habría ocurrido en un marco de negociación como principal estrategia política. 
No obstante los acuerdos en torno a esta premisa, nos encontramos aún con algunos puntos ciegos acerca de la sintaxis del vínculo entre la geopolítica local y aquella simbolizada en los intereses imperiales. Algunas propuestas para la macroregión ponen el acento en el factor ideológico/sagrado para explicar la apropiación de los paisajes locales. De acuerdo a esto, el componente sagrado que impregna las montañas andinas, por ejemplo, habría funcionado como un capital mayor para los incas, que encontraron en estos espacios una potente estrategia para legitimar y consolidar su poder. Las propuestas para el vecino altiplano boliviano, sugieren que la incorporación de los territorios obedece a la mecánica de la "conquista ritual" (Cruz 2009: 71) a partir de las wakas locales, cada una asociada a una jurisdicción, por lo que el control de estos espacios habría tenido un importante impacto en el vínculo con las poblaciones del lugar. Algo similar es indicado acerca del territorio chileno, en donde la veneración de estos lugares de privilegio en relación al valle donde estaban asentadas las poblaciones locales, es interpretado como una representación contundente de la imagen de poder encarnada la figura del estado (Pavlovic y Rosende 2010; Pavlovic et al. 2019). Estos enfoques, sostenidos fuertemente en la noción de paisaje y en el componente religioso o sagrado de los mismos, desafían el protagonismo que tuvo hasta hace algunas décadas el factor económico, representado en el acceso a los recursos -naturales o humanos-, que primó en las explicaciones sobre la presencia incaica en los territorios más meridionales (González 1980; Lorandi 1988; Raffino2007).

El énfasis puesto en las relaciones entre entidades en el marco de un sistema de referencia situado en la síntesis del espacio físico y el espacio social (Criado Boado 2017), desde el cual entender la tradicional noción de conquista incaica, nos lleva a discutir dos ideas medulares para el abordaje de los paisajes del pasado: la integración y la articulación (Sánchez Palencia et al. 1996). Ambos conceptos pueden analizarse a partir de un recorte sincrónico, situado sobre la variable espacio, o diacrónico, apelando a la constante tiempo o, como el caso que nos convoca, a partir de ambos. Por otro lado, no hay una única manera de resolver su análisis, el que va a depender fundamentalmente de las posibilidades y potencialidades del correlato empírico del que dispongamos.

Sobre esta base, la propuesta del trabajo es analizar la articulación e integración entre instalaciones asignadas al Período Tardío (siglos X-XIV) e Inca (siglos XIV-XV) en el sector norte del Valle Calchaquí. Para eso trabajaremos desde la noción de paisaje previa, atendiendo a la visibilidad como una variable de medida del entorno que permite una aproximación a las lógicas organizativas con la que se manejan las sociedades. Como tal, la visibilidad depende tanto de la información sensorial de los propios individuos como de la memoria social que interviene en el acto cognitivo, es decir, lleva implícito el componente cultural pero también histórico (Witcher 1999). 


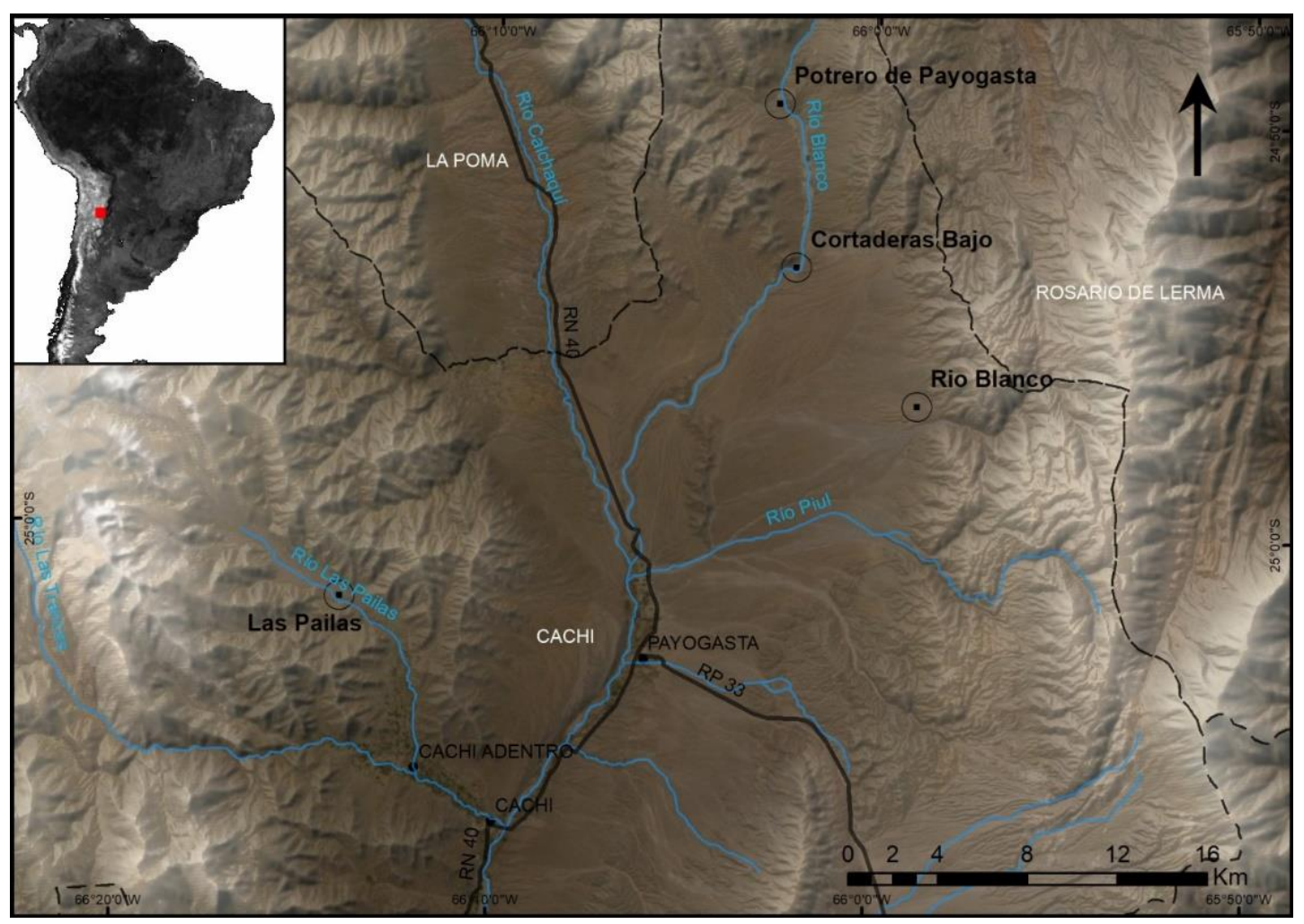

Figura 1. Área de estudio

\section{Los sitios y sus contextos}

En virtud de lo anterior, partimos de la hipótesis de que los sitios con una arquitectura incaica se localizarían en sectores con un mejor manejo visual del entorno, en particular si se los compara con los que fueron ocupados por las poblaciones locales durante los momentos previos (Hyslop 1990).

Para analizar esto, contrastamos dos sitios con ocupación tardía, que se destacan por la gran extensión de campos agrícolas y estructuras de irrigación: Río Blanco y Las Pailas (Páez y López 2019) con otros dos con clara arquitectura incaica que han sido trabajados por otros investigadores: Potrero de Payogasta y Cortaderas Bajo (Acuto, 1999; D'Altroy et al. 2000; De Marrais 2013). Hacemos la salvedad de que si bien se han encontrado elementos que podrían sugerir que el sitio Las Pailas fue incorporado a la esfera de control incaico (Páez y Giovannetti 2014), tanto la elección del lugar como su patrón arquitectónico responde a las características propias de la ocupación indígena local.

Para la elección de los sitios a comparar se tomaron en cuenta dos criterios fundamentales: la localización geográfica y la presencia de arquitectura supradoméstica. En relación al primero, en los cuatro casos se trata de altitudes superiores a los $2500 \mathrm{msnm}$, a los fines de que la condición altitudinal no represente per se un factor intrínseco de variabilidad (Tabla 1). En cuanto a la localización cardinal, dos de los sitios se ubican hacia el oeste del Valle, Potrero de Payogasta hacia el suroeste y Las Pailas hacia el este. La posibilidad de trabajar con espacios que, desde el punto de vista 
público o productivo, enmarquen relaciones comunitarias cobra relevancia desde dos aspectos. Por un lado, implica que esos lugares fueron percibidos y habitados por una parte importante de la población bajo nociones compartidas acerca del territorio. No obstante, además de estos aspectos relacionados con la pertenencia o la identidad, también de juegan elementos de orden histórico, por el cual esos lugares fueron elegidos sobre la base de la experiencia y significado que tuvieron, y que se cimentó en la memoria de sus pobladores.

Los dos sitios tardíos tienen la singularidad de oponerse geográficamente, si bien los registros altitudinales son concordantes. Ambos espacios presentan una amplia extensión de cuadros de cultivo y estructuras de riego (Páez y Giovannetti 2014; Páez y López 2016, 2019), que permitieron una producción agrícola a gran escala, en la que habría participado un importante número de trabajadores. Para el caso de Las Pailas, contiguo al sector agrícola se encuentra un conjunto de recintos irregulares que conformó un poblado semiconglomerado (Tarragó 1977; Tarragó y De Lorenzi 1976) vinculado a la residencia y al procesamiento de los productos, a juzgar por la abundancia de morteros móviles en el interior de los recintos (Páez et al. 2012), además de elementos arquitectónicos con un consistente valor ritual (Páez y Marinangeli 2016; Páez et al. 2014, 2016). Río Blanco ha sido muy poco investigado y actualmente se encuentra en terrenos privados utilizados para el cultivo de vid de altura, por lo que los datos disponibles son mucho menores. Esta condición representa un limitante para arribar a su organización espacial dado que, si había un conglomerado habitacional similar al de Las Pailas, no se ha conservado. Al margen de ello, la arquitectura de los campos, así como los rasgos generales de la red hidráulica permiten trazar paralelismos entre uno y otro espacio de cultivo.

La vinculación de Potrero de Payogasta y Cortaderas a la política incaica aflora, en particular, a partir de la arquitectura, que se relaciona con una función administrativa (Acuto 1999; D'Altroy et al. 2000). Cortaderas Bajo es una parte de un conjunto arquitectónico mayor. Está constituido por una fortaleza incaica en la cima de un cerro bajo, y dos sectores inmediatamente por debajo. Uno de ellos se compone de dos kanchas relacionadas con las actividades del Estado, en tanto el otro presenta recintos habitacionales donde habrían tenido lugar actividades domésticas. En el medio se ubica una plaza, rodeada por un muro perimetral (Acuto y Gifford 2007). De acuerdo a los autores, la arquitectura de este sector es netamente incaica. Potrero de Payogasta, por su parte, tiene una organización del espacio planificada, que reproduce las prerrogativas cusqueñas (D'Altroy et al. 2000). Puede reconocerse una plaza, un ushnu y una kallanka, asociados a recintos identificados como kanchas, en donde habrían tenido lugar actividades de carácter público congregativas y ceremoniales. Este complejo está acompañado también por recintos asociados a patios y estructuras de almacenamiento, que dan cuenta de que en el sitio también se desarrollaron actividades productivas y residenciales (D'Altroy et al. 2000; Earle 1994). 


\begin{tabular}{|c|c|c|c|}
\hline Sitio arqueológico & Ocupación & Funcionalidad & $\begin{array}{c}\text { Altitud } \\
\text { (msnm) }\end{array}$ \\
\hline Las Pailas & Indígena local & Productivo/residencial & 3087 \\
\hline Río Blanco & Indígena local & Productivo & 2954 \\
\hline $\begin{array}{c}\text { Potrero de Payogasta } \\
\text { (D'Altroy et al. 2000) }\end{array}$ & Inca & $\begin{array}{c}\text { Administrativo/ } \\
\text { ceremonial/residencial }\end{array}$ & 2889 \\
\hline $\begin{array}{c}\text { Cortaderas Bajo } \\
\text { (Acuto 1999) }\end{array}$ & Inca & $\begin{array}{c}\text { Actividades estatales/ } \\
\text { residencial }\end{array}$ & 2759 \\
\hline
\end{tabular}

Tabla 1. Sitios arqueológicos incorporados al estudio

\section{El análisis de visibilidad}

El estudio de visibilidad planteado en función de los objetivos comprende un análisis de cuencas visuales acumuladas y puntos de observador. Los análisis y modelos basados en programas informáticos y algoritmos matemáticos, de la mano con los llamados Sistemas de Información Geográfica (SIG), y la creciente disponibilidad de productos derivados de imágenes de sensores remotos, permiten abordar la percepción visual como aporte de interés para el estudio del paisaje arqueológico, tanto desde la exploración teórica como en el campo de la metodología (Criado Boado 1991, 1993, 1999; García Sanjuán 2004; Lock y Harris 1996; Llobera 2003, 2006, 2007; Tilley 1994; Wheatley 2004; Wheatley y Gillings 2000, 2013; Wood 1996; Zamora Merchán 2006, 2013). Estas tecnologías ofrecen un amplio abanico de opciones para realizar cálculos y estimaciones acerca del comportamiento de variables a partir de su información geoespacial asociada (Wheatley y Gillings 2013).

La estimación de cuencas visuales es una de ellas, referida al cálculo de la superficie del terreno potencialmente visible desde un punto determinado, utilizando como insumo un Modelo Digital de Elevación (MDE). En el caso de este trabajo se utiliza un modelo raster o matriz de valores, que codifica para cada celda un valor de altitud del terreno. De esta manera, conociendo el dato de localización geoespacial de un punto de interés, la herramienta permite calcular qué puede verse y qué no desde este punto en base al cálculo de ángulos verticales a partir del MDE.

Una variante del cálculo de cuencas visuales son las llamadas cuencas visuales acumuladas, que estiman la superficie visible desde varios puntos de observador en lugar de solo uno. El valor de aquellos pixeles que resultan visibles desde más de un punto se acumulan mediante un conteo de los puntos de observador de forma que las celdas en la matriz raster resultante pueden asumir tantos valores como puntos de observador se consideren. Es decir, mientras un análisis de cuenca visual desde un punto de observación devuelve una capa matricial binaria (cuyos valores pueden ser 0 o 1), un análisis de cuenca visual acumulada entrega una capa matricial cuyas celdas pueden asumir todos los valores desde 0 hasta el máximo de puntos de observación 
incluidos en el análisis. El resultado permite entonces conocer las superficies del terreno que son visibles desde una mayor o menor cantidad de puntos de observador, habilitando ulteriormente a realizar evaluaciones de mayor precisión en base a los resultados del análisis (Zamora Merchán 2006).

Vale aclarar que un análisis de visibilidad propiamente dicho no se agota en el cálculo de la superficie potencialmente visible del terreno desde un punto dado, constituyendo esta estimación una mera fuente de información que puede aportar a profundizar algún aspecto más amplio del estudio de la visibilidad en el pasado. El hecho de que un ítem en el paisaje sea potencialmente visible no implica que haya habido un interés o una voluntad por dirigir la mirada hacia él, y tampoco implica que haya existido una intención por hacer de tal elemento un objeto visible desde tal o cual lugar, o en caso contrario, de ocultarlo de la visión (Criado Boado 1993).

Los puntos utilizados para realizar el cálculo de cuencas visuales acumuladas fueron dispuestos de forma equidistante, distribuidos uniformemente guardando una distancia de $100 \mathrm{~m}$ entre uno y otro en el área de cada uno de los sitios referidos en la Tabla 1, aumentando así la representatividad y precisión de la estimación. Por otra parte, sobre los datos precedentes se incorporó la Intervisibilidad como variable de análisis, que constituye una observación acerca de si dos o más puntos son visibles el uno desde el otro y viceversa, en este caso referidos específicamente a las relaciones visuales entre los sitios arqueológicos mencionados.

Estos análisis fueron realizados mediante el software ArcMap 10.2.2 (ArcGIS ESRI) utilizando la herramienta "Viewshed". Como insumo se utilizó un Modelo Digital de Elevación del proyecto MDE-Ar v2.01 que distribuye el IGN (Instituto Geográfico Nacional). Este producto tiene una resolución espacial de $30 \mathrm{~m}$ (extensión de superficie que representa cada pixel sobre el terreno) y una precisión vertical de aproximadamente $2 \mathrm{~m}$, requiriéndose para este trabajo un total de cuatro productos correspondientes a las cartas de escala 1:100.000 que cubren el área de estudio, creando con ellos un mosaico mediante ArcMap. Se estableció una altura del punto de observador desde el suelo de 1,5 m que constituye un promedio de la altura de observación de acuerdo a la estatura de los sujetos. Dadas las características atmosféricas en la zona, así como la vegetación local en el periodo tomado para el análisis, no ha sido necesario recurrir a datos paleoclimáticos o paleoambientales para ajustar las variables.

Por otra parte, las mencionadas condiciones atmosféricas y atributos generales del ambiente en lo que respecta al acceso visual sobre el entorno hacen que, a la hora de analizar la cobertura visual desde distintos sitios sobre grandes áreas, no resulte pertinente establecer un límite de alcance visual, que queda entonces comprendido dentro del área de estudio establecida. 


\section{Distancia e intervisibilidad}

Para el caso de Río Blanco, se dispuso un total de 168 puntos para el cálculo de la cuenca visual acumulada, al tratarse de un espacio agrícola de grandes dimensiones. De estos puntos, entre 100 y 130 (colores amarillo y naranja en la Figura 2a) tienen acceso visual al fondo de valle en la zona próxima a las márgenes del Rio Calchaquí, mientras la zona sur de piedemonte sólo es visible desde un número de puntos que oscilan entre los 20 y 100 aproximadamente (correspondientes a los colores azules y verdes en la Figura 2a). Las zonas altas occidentales y septentrionales del área de estudio son visibles desde la totalidad de los puntos establecidos para la estimación de la cuenca (color rojo).

La causa de esta segregación en la frecuencia de puntos desde los cuales hay acceso visual a las distintas subáreas responde al emplazamiento del sitio, ubicado en una zona de cambio de pendientes. Los puntos más próximos a las laderas poseen mayor altura, pero se encuentran encajonados entre estructuras rocosas propias de esta transición, lo cual reduce su ángulo de visión periférica impidiendo el acceso visual a las zonas de piedemonte, tanto al norte como al sur del sitio. En cambio, los puntos más próximos a las planicies encuentran una menor cantidad de obstáculos y un acceso visual más allanado sobre una mayor variedad de subáreas.

De este modo, la cuenca visual de Río Blanco abarca gran parte del fondo de Valle, salvo la superficie que aparece obstruida por los cerrillos, algunos segmentos próximos del piedemonte de la ladera oriental, y las diversas cumbres y laderas occidentales del valle. El acceso visual a las zonas bajas circundantes al río Calchaquí y las zonas altas de la ladera occidental es privilegiado, incluyendo el Nevado de Cachi, destacado en el paisaje ritual local (Leibowicz y Jacob 2014; Vitry 2007).

Aquella correspondiente a los puntos de observación de Las Pailas, por su parte, se encuentra bastante restringida en superficie debido a que el sitio está rodeado por cerros a excepción de su extremo sur-este. En comparación con la cuenca visual de Rio Blanco, Las Pailas presenta un resultado más homogéneo: de los 143 puntos dispuestos para realizar la estimación, la gran mayoría cubre áreas muy semejantes. Se puede hacer la salvedad de la zona sur del piedemonte y de las tierras altas orientales, que son las únicas subáreas visibles por fuera del ámbito propio del sitio y de las cumbres más elevadas de occidente. Dado que el sitio tiene pendientes muy marcadas presentando un incremento en las altitudes en sentido SE-NW, conforme los puntos se encuentren dispuestos de forma más próxima al extremo NW tendrán mayor acceso visual sobre la zona sur de piedemonte (color azul y verde en la Figura 2b). Es decir, esta zona sólo es visible desde unos 15 a 70 puntos de observación de manera gradual, conforme sea mayor la altitud de los mismos (Figura 2b). Aunque el resultado para Las Pailas muestra algunos solapamientos con la cuenca visual acumulada del sitio Rio Blanco, en términos generales ambas cuencas cubren áreas distintas, los sitios no son intervisibles, y de hecho en algunos sectores las cuencas se tocan en las divisorias de aguas de los cerros. 

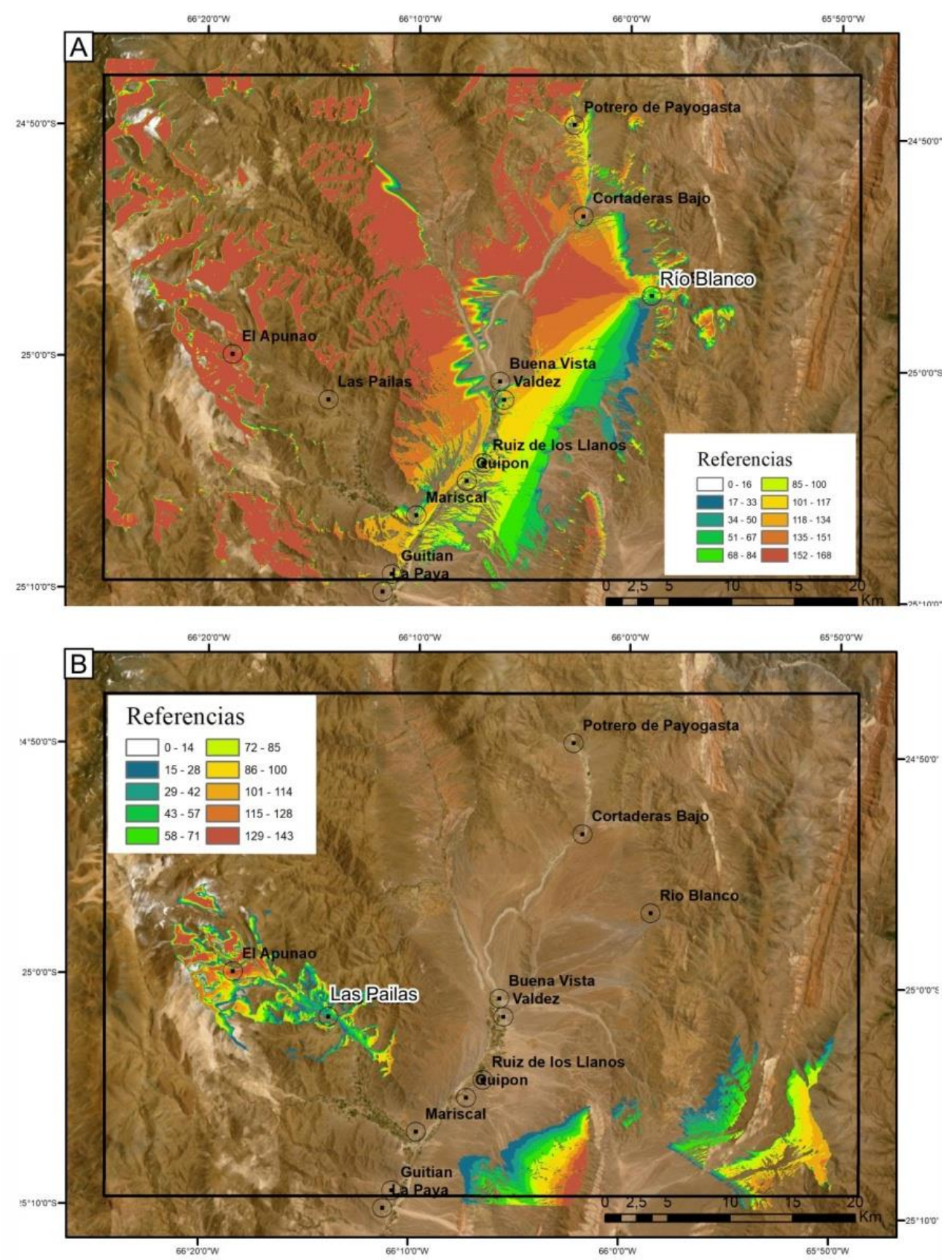

Figura 2. Cuenca visual acumulada delos sitios A) Rio Blanco y B) Las Pailas.

En lo que respecta a las cuencas de Potrero de Payogasta y Cortaderas Bajo, abarcan las áreas de altura de las laderas orientales. Sin embargo es notorio que desde el primero hay una buena visibilidad de las zonas elevadas septentrionales que no son visibles desde Cortaderas Bajo, mientras que desde este último ocurre lo propio para el segmento norte de las cumbres occidentales que no son visibles desde Potrero de Payogasta. Ambos sitios comparten áreas de visibilidad en la zona central de piedemonte al este del valle y ninguna de las dos presenta buen acceso visual al fondo de valle o la zona sur del área de estudio (Figura 3). La visibilidad parece concentrarse en zonas elevadas, presentándose escasa o nula en relación a las márgenes del río Calchaquí, donde se ubican una gran cantidad de sitios arqueológicos con ocupación local durante el Segundo Milenio. 
Particularmente el sitio Potrero de Payogasta presenta una cuenca visual muy restringida a las zonas altas próximas al mismo, aun cuando se utilizaron un total de 67 puntos distribuidos uniformemente en toda la cobertura del sitio, de los cuales sólo entre siete y 30 (colores azul y verde en la Figura 3a) tienen acceso visual sobre la zona de piedemonte mientras que casi la totalidad lo tiene sobre las zonas elevadas.
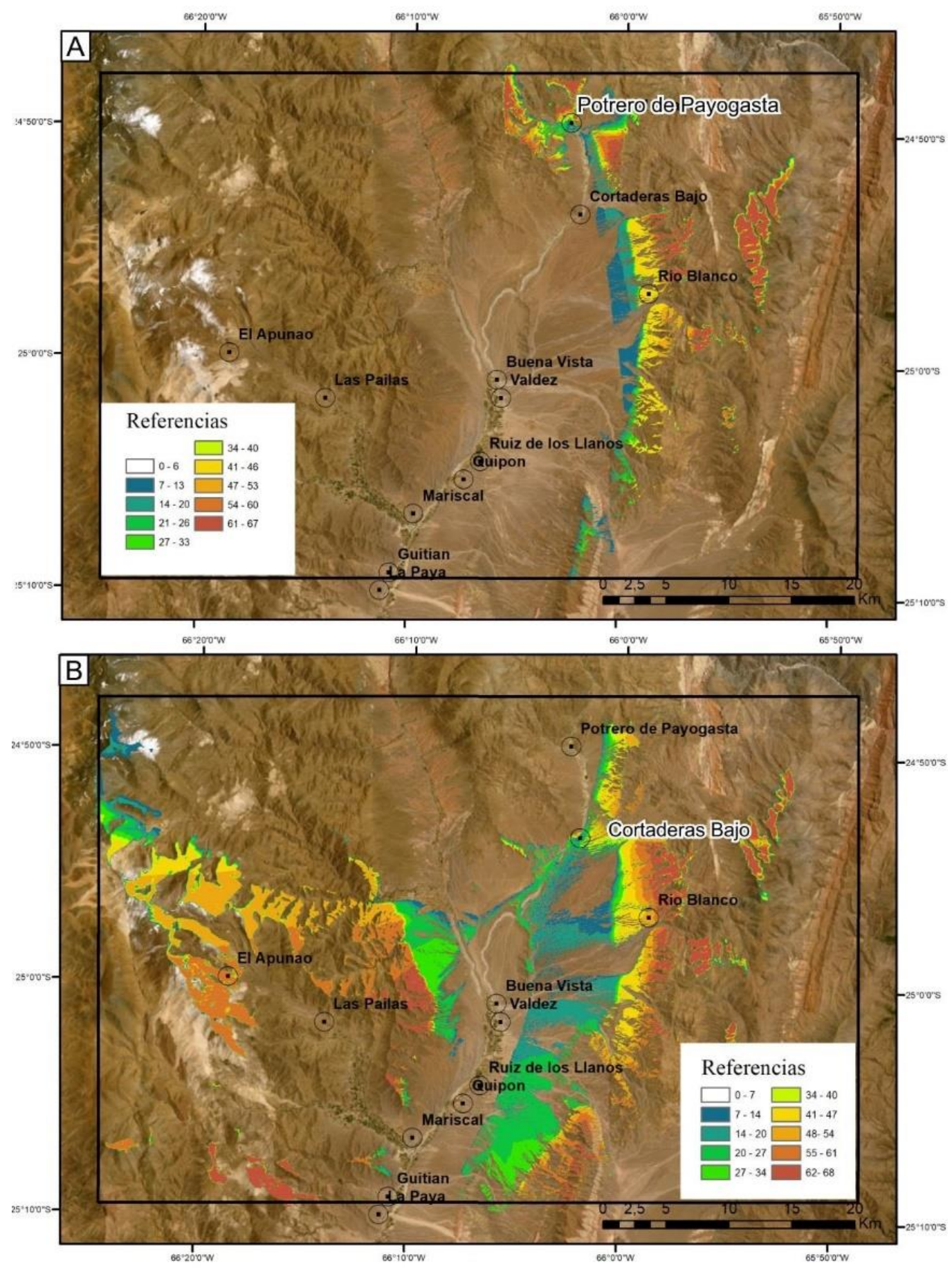

Figura 3. Cuenca visual acumulada de los sitios A) Potrero de Payogasta y B) Cortaderas Bajo.

En lo que respecta a la cuenca visual acumulada del sitio Cortaderas Bajo, la diversidad en las áreas visualmente accesibles es mayor, ya que casi la totalidad de los 68 puntos utilizados para realizar el cálculo abarcan también parte de las zonas 
elevadas tanto occidentales como orientales (colores naranja y rojo en la Figura $3 b$ ), mientras que solo unos siete a 30 de estos puntos (colores azul y verde de la Figura $3 b$ ) presenta acceso visual sobre el piedemonte -un sector de visibilidad nula desde el sitio Potrero de Payogasta-. Por otro lado, no existe intervisibilidad entre estos dos sitios, lo que resulta llamativo considerando su concordancia cronológica y articulación en términos de la política incaica.

Finalmente, y sobre la base de los resultados expuestos en las Figuras 2 y Figura 3, se realizó un análisis de intervisibilidad entre los cuatro sitios descriptos y algunos de los sitios más relevantes del Segundo Milenio prehispánico ubicados en cercanías del río Calchaquí. Se incluyeron los sitios Buena Vista, Valdez, Ruiz de los Llanos, Quipón, Mariscal, Tero, La Paya y Guitián (Baldini y De Feo 2000; De Marrais 1997, 2001; D'Altroy et al. 2000; Sprovieri 2005; Tarragó y De Lorenzi 1976; Williams y D'Altroy 1998).

Se pudo observar que la visibilidad entre los sitios incaicos y el fondo de valle es prácticamente nula (Figura 3), al igual que ocurre con el sitio Las Pailas (Figura 2). Por el contrario, la visibilidad sobre los sitios del fondo de valle desde el sitio Rio Blanco es muy buena, de forma tal que todos los sitios citados salvo Buena Vista (por la obstrucción de Los Cerrillos), La Paya y Guitián ubicados en el extremo sur del área de estudio, son visibles desde más de 100 de los puntos distribuidos en Rio Blanco (áreas amarilla y naranja en la Figura 2a). Estas observaciones permiten estimar una buena intervisibilidad entre las dos áreas, que se continúa hacia el norte, siguiendo el trazado del río Calchaquí.

\section{Discusión y conclusiones}

Del análisis de los datos se desprende, en primera instancia, que el punto de observación con mayor dominio visual del Valle es Río Blanco, ocupado durante el Período Tardío con una arquitectura vinculada a la función agrícola. A excepción del sitio Las Pailas, tanto aquellos ubicados en el fondo de Valle, como las áreas intermedias y los dos sitios incaicos, Cortaderas Bajo y Potrero de Payogasta, son visibles desde aquí.

La comparación entre las cuencas acumuladas correspondientes a cada uno de los cuatro sitios indica una preeminencia visual de Río Blanco por sobre los otros y, si bien Cortaderas también tiene un control importante del paisaje, es significativamente menor al del sitio agrícola. Tanto desde este sitio como de Potrero de Payogasta se puede observar Río Blanco, pero no Las Pailas, y lo que resulta aún más llamativo es que Cortaderas Bajo y Potrero de Payogasta no son intervisibles, ni desde allí es posible observar el fondo de Valle. Otro aspecto a remarcar es que Potrero de Payogasta y Las Pailas tienen cuencas visuales restringidas, concentradas en las partes altas: en el caso del primero en dirección sur, en tanto que en el segundo, hacia el sureste. 
Esto aporta dos elementos centrales a discutir: por un lado, el grado de integración de los sitios en el paisaje visual y por otro, de qué manera la visibilidad se puede vincular con la idea del control o dominio del entorno social para el área de estudio. En relación a lo primero, los sitios del fondo de Valle incorporados al análisis, y que se asignan a ocupaciones indígenas locales del Período Tardío, se articulan visualmente con el punto de observación de Río Blanco, no siendo visibles desde y hacia Potrero de Payogasta y Cortaderas Bajo. Un análisis discriminado de las cuencas visuales de cada uno de los sitios ubicados en proximidades del río Calchaquí nos permitiría mayores precisiones al respecto, por ejemplo, desde qué localizaciones precisas es posible observar el sitio agrícola tardío. Sin embargo, la falta de intervisibilidad con los sitios incaicos es un dato relevante per se, porque no sólo significa que desde los sitios incaicos no se veía qué es lo que ocurría abajo, sino que estos símbolo arquitectónicos del poder del Estado tampoco eran visibles desde las residencias de los pobladores locales. El caso de Las Pailas es particular porque está al otro lado del Valle. No obstante esto, su encajonamiento es un condicionante a considerar en relación a la visibilidad, que se limita al espacio más inmediato. Es probable que en la elección de este lugar para el cultivo hayan tenido un rol preponderante las características geográficas del sector en términos del suelo, y la disponibilidad permanente de agua a través de los ríos Peñas Blancas y Potrerillos como contrapartida a las condiciones de semiaridez de la región (Páez y López 2016, 2019).

El segundo aspecto a analizar tiene que ver con que, contrariamente a lo esperado, el mayor control visual del entorno aparece asociado a espacios comunales de producción agrícola, en contraposición a los sitios vinculados al ejercicio de la función administrativa estatal que, como mencionamos, tienen un carácter más bien disruptivo en el paisaje. El manejo visual de Potrero de Payogasta es hacia las mayores altitudes, no hacia el fondo de Valle, y en particular hacia el sur. Si bien hoy sabemos que el sitio no se estableció en un entorno deshabitado, sino que a su alrededor había una importante cantidad de sitios indígenas tardíos (Vitry et al. 2007) -algunos de los cuales podrían entrar dentro del abanico de visibilidad que presentamos-, no parece haber dudas acerca de que la integración visual con los lugares de residencia de la población tardía no sería el criterio elegido para el establecimiento del sitio. En cambio, esta ubicación sí garantizaría la comunicación del Estado inca a través del sistema vial (Vitry y Soria 2007), además del control de las vías de ingreso al Valle (Cabral Ortiz, com. pers. 2020).

Finalmente, y si bien no se incorporó dentro del análisis, uno de los puntos de mayor visibilidad desde y hacia las altitudes menores, intermedias y altas, es el nevado de Cachi (Leibowicz y Jacob 2014; Vitry 2007), cuya materializad estimula la idea de que sería la principal waka dentro del entorno de estudio. Su altitud lo convierte en un hito privilegiado del paisaje. En este punto es interesante retomar la comparación, en términos de visibilidad, con el sitio Río Blanco donde, con una altitud significativamente menor se logra un abanico de observación comparable en términos relativos. Esto pone en relieve el valor espacialmente estratégico de Río Blanco, y sus posibilidades de integrar visualmente el paisaje del Valle. 
A partir de lo anterior y a manera de conclusión del trabajo pero a la vez, condición de apertura de nuevos interrogantes, vale preguntarnos acerca de cuán oportuno sea pensar el paisaje calchaquí más allá de los axiomas creados para otras geografías (y concomitantes historias). Aquí la visibilidad parece asociarse, no con la idea de hegemonía y/o dominio del medio, sino más bien a una condición de integración y articulación, que está bien representada en la cartografía de los momentos tardíos. En estos términos, en la construcción de lo colectivo o lo comunitario, la visibilidad habría jugado un papel importante, probablemente garantizando los términos de la interacción. Esto de ninguna manera pone en cuestión las condiciones de la presencia incaica en el ámbito calchaquí, la que al menos a partir de los dos sitios estudiados ha tenido un carácter notablemente disruptivo si se la compara con la organización del paisaje indígena local.

Agradecimientos: A la comunidad de Cachi y Payogasta, a las autoridades del Museo Antropológico de Salta y el Museo Pío Pablo Díaz de Cachi, a la Comunidad Diaguita Kallchaquí de Las Pailas, al Lic. Jorge Cabral Ortiz por el intercambio de conocimientos y a los compañeros del equipo de trabajo por su colaboración en los trabajos de campo. A los revisores anónimos, que nos ayudaron a mejorar la versión original del manuscrito y al Consejo Nacional de Investigaciones Científicas y Técnicas y la Agencia Nacional de Investigaciones Científicas que facilitó el financiamiento de las tareas de investigación. La responsabilidad por lo expresado, no obstante, es exclusiva de los autores.

\section{Notas}

${ }^{1}$ Estos productos son creados mediante una combinación de datos capturados por las misiones satelitales SRTM y ALOS, cuya referencia vertical fue ajustado al Sistema de Referencia Vertical Nacional (SRVN16).

https://www.ign.gob.ar/NuestrasActividades/Geodesia/ModeloDigitalElevaciones/ Introduccion

\section{Bibliografía citada}

Acuto, F.

1999 Paisaje y dominación: La constitución del espacio social en el Imperio Inka. Sed non Satiata. Teoría Social en la Arqueología Latinoamericana Contemporánea, (ed. por A. Zarankin y F. Acuto), pp. 33-75. Ediciones Del Tridente, Buenos Aires.

Acuto, F. y C. Gifford

2007 Lugar, arquitectura y narrativas de poder. Experiencia y percepción en los centros inkas de los Andes del Sur. Arqueología Suramericana 3 (2): 135-161.

Baldini, L. y C. De Feo

2000 Hacia un modelo de ocupación del Valle Calchaquí Central (Salta) durante los Desarrollos Regionales. Relaciones de la Sociedad Argentina de Antropología XXV: 75-98. 
Criado Boado, F.

1991 Construcción social del espacio y reconstrucción arqueológica del paisaje. Boletín de antropología americana (24): 5-29.

1993 Visibilidad e interpretación del registro arqueológico. Trabajos de prehistoria, 50: 39-56.

1999 Del terreno al espacio: planteamientos y perspectivas para la arqueología del paisaje. Universidad de Santiago de Compostela, España.

2017 A paisaxe como enxeñería inversa da materia e da memoria. Paisaxe $e$ Patrimonio, un percorrido polo territorio a través do Arquivo de Galicia, (ed. por C. García García y M. García Miraz), pp. 147-57. Xunta de Galicia, Consellería de Educación e Cultura, Arquivo de Galicia, Santiago de Compostela.

Cruz, P.

2009 Huacas olvidadas y cerros santos: Apuntes metodológicos sobre la cartografía sagrada en los Andes del sur de Bolivia, Estudios Atacameños 38(1): 55-74.

D’Altroy, T., A. M. Lorandi, V. Williams, M. Calderari, C. Hastorf, E. DeMarrais, M. Hagstrum

2000 Inka rule in the Northern Calchaqui Valley, Argentina, Journal of field Archaeology 27: 1-26.

DeMarrais, E.

1997 Materialization, ideology, and power: The development of centralized authority among the pre-Hispanic polities of the Valle Calchaquí, Argentina Doctoral dissertation. University of California, Los Angeles.

2001 La Arqueología del norte del valle Calchaquí. Historia Argentina Prehispánica, (ed. por Berberian, E. y A. Nielsen), Tomo 1, pp. 310-330. Editorial Brujas, Córdoba.

2013 Colonización interna, cultura material y poder en el Imperio Inca. Relaciones de la Sociedad Argentina de Antropología XXXVIII (2): 351-376.

Díaz, P. P

1983 Sitios arqueológicos del Valle Calchaquí, Estudios Arqueológicos 3-4.

Earle, T.

1994 Wealth Finance in the Inka Empire: Evidence from the Calchaqui Valley, Argentina, American Antiquity 59(3): 443.

García Sanjuán, L.

2004 La prospección arqueológica de superficie y los SIG. Actas del I Encuentro Internacional de Informática Aplicada a la Investigación y la Gestión Arqueológicas, pp. 185-210. 
González, A. R

1980 Patrones de asentamiento incaico en una provincia marginal del imperio; implicancias socio-culturales, Relaciones de la Sociedad Argentina de Antropología XIV, 1: 63-82.

Hyslop, J.

1990 Inka Settlement Planning. University of Texas Press, Austin.

Lorandi, A. M.

1988 Los diaguitas y el Tawantinsuyu: una hipótesis del conflicto. La frontera del Estado inca. Actas del 45.o Congreso Internacional de Americanistas, (ed. por T. D. Dillehay y P. J. Netherly), pp. 235-259. BAR International Series 422, Oxford.

Leibowicz, I. y C. Jacob

2014 Ceremonias en la tormenta. Ritual Inka en el Nevado de Cachi, Estudios. Antropológía Historia, Nueva Serie 2: 27-48.

Llobera, M.

2003 Extending GIS-based visual analysis: the concept of visualscapes. International Journal of Geographical Information Science 17(1): 25-48.

2006 Arqueología del paisaje en el siglo XXI: reflexiones sobre el uso de los SIG y modelos matemáticos. La aplicación de los SIG en la Arqueología del Paisaje, (ed. por Grau Mira, I.), pp. 109-124. Universidad de Alicante, Alicante.

2007 Reconstructing visual landscapes. World Archaeology 39(1): 51-69.

Lock, G. y T. Harris

1996 Danebury revisited an English Iron Age hillfort in a digital landscape. Anthropology, Space, and Geographic Information Systems, (ed. por Aldenderfer, M. y Maschner, H.), pp. 214-240. Oxford University Press, New York.

Páez, M. C. y M. Giovannetti

2014 Canales aéreos y subterráneos de Las Pailas (Cachi, Salta): Aproximaciones al estudio de la red hidráulica. Estudios Antropología-Historia, Nueva Serie 2: 20-35.

Páez, M.C. y L. López

2016 La tecnología hidráulica del Valle Calchaquí Norte (Cachi, Salta). Actas del XIX Congreso Nacional de Arqueología Argentina, pp. 1977-1985. Universidad Nacional de Tucumán.

2019 Irrigation canals from the Calchaqui valley (province of Salta, Argentina). Journal of Archaeological Science: Reports 27, https://doi.org/10.1016/j.jasrep.2019.101989 
Páez, M. C. y G. Marinangeli

2016 Huancas and rituals of fertility in the farming landscape of the northern Calchaquí Valley (Salta, Argentina). Latin American Antiquity 27(1): 115-31.

Páez, M. C., M. Giovannetti y R. Raffino

2012 Las Pailas. Nuevos aportes para la comprensión de la agricultura prehispánica en el Valle Calchaquí Norte (provincia de Salta), Revista Española de Antropología Americana 42 (2): 339-357.

Páez, M. C., V. Lynch e Y. Besa

2014 Espacios sagrados en el mundo andino: excavación de una huanca en Las Pailas (Cachi, Salta, Argentina). Revista Española de Antropología Americana 44 (1):275-284.

Páez, M. C., Marinangeli, G. y M. E. Prieto

2016 Significación e historicidad en el paisaje campesino del valle Calchaquí Norte (provincia de Salta, Argentina). Historia Agraria 68: 137-156.

Pavlovic, D. y E. Rosende

2010 Más cerca de las Wakas: la ocupación de cerros de mediana y baja altura durante el período Tardío en la cuenca superior del río Aconcagua. Actas del XVII Congreso Nacional de Arqueología Argentina Tomo III-IV, 1279-1284.

Pavlovic, D., R. Sánchez, D. Pascual, A. Martínez, C. Cortés, C. Dávila y N. La Mura 2019 Rituales de la vida y de la muerte: dinámicas de interacción entre el Tawantinsuyu y las poblaciones locales en la cuenca del Maipo-Mapocho, Chile central, Estudios atacameños 63: 43-80.

Pollard, G.

1983 The Prehistory of NW Argentina: The Calchaquí Valley Project, 1977-1981, Journal of Field Archaeology 10: 11-32.

Raffino, R.

2007 Poblaciones indígenas en Argentina. Urbanismo y proceso social precolombino. Emecé, Buenos Aires.

Rivolta, M. C., V. Seldes, J. Rodríguez, L. Yazlle y M.F. Zigarán.

2007 Las Sociedades del Formativo en Cachi (Salta, Argentina). Aproximaciones al entorno de Salvatierra. Actas del XVI Congreso Nacional de Arqueología Argentina II: 333336.

Rodríguez, J. y M. C. Rivolta

2010 La producción alfarera en los sitios formativos del valle Calchaquí norte: aproximaciones al estudio de espacios domésticos y funerarios. Actas del XVII Congreso Nacional de Arqueología Argentina I: 171-176. 
Rivolta, M. C. y J. Cabral Ortiz

2017 El espacio doméstico en las ocupaciones aldeanas del Valle Calchaquí Norte (Salta, Argentina). Arqueología Iberoamericana 36: 66-78.

Sánchez-Palencia, F., M. D. Fernández-Posse, J. Fernández Manzano, A. Orejas, Y. Álvárez González, L. López González y L. Pérez García

1996 Las Zonas Arqueológicas como paisajes culturales: el parque arqueológico de Las Médulas (León). Complutum 6(11): 383-403.

Seldes, V. y E. I. Baffi

2016 Alimentación en la prehistoria. El caso de valle Calchaquí, Salta, Argentina. RUNA, Archivo Para Las Ciencias Del Hombre 37(2): 79-95.

Sprovieri, M.

2005 Manejo de recursos y producción lítica en sociedades tardías del Valle Calchaquí (Salta). Tesis de Licenciatura, Facultad de Filosofía y Letras, Universidad de Buenos Aires.

Tarragó, M.

1977 La localidad arqueológica de Las Pailas, provincia de Salta, Argentina. Actas del VII Congreso de Arqueología de Chile Vol. II, pp. 499-517. Chile.

1978 Paleoecology of the Calchaquí Valley, Salta Province, Argentina. Advances in Andean Archaeology, (ed. por D. Browman), pp. 485-512. Mouton, La Haya.

1995 Desarrollo regional en Yocavil: una estrategia de investigación. Actas del XIII Congreso Nacional de Arqueología Chilena, Hombre y Desierto 9: 225-236.

1996 El Formativo en el Noroeste argentino y el alto valle Calchaquí. Revista del Museo de Historia de San Rafael 23 (1/4): 103-119.

Tarragó, M. y P. P. Díaz

1972 Sitios arqueológicos del Valle Calchaquí. Estudios de Arqueología 2: 49-62.

1977 Sitios arqueológicos del valle Calchaquí. Estudios de Arqueología 2: 63-71

Tarragó, M y M. De Lorenzi

1976 Arqueología del Valle Calchaquí. Etnía 23-24: 1-35.

Tilley, C.

1994 A phenomenology of landscape: places, paths, and monuments. Berg, Oxford.

Vitry, C.

2007 Caminos rituales y montañas sagradas. Estudio de la vialidad inka en el Nevado de Chañi, Argentina. Boletín del Museo Chileno de Arte Precolombino 12(2): 69-84. 
Vitry, C. y S. Soria

2007 Sistema de asentamiento prehispánico en la sierra meridional de Chañi (Salta, Argentina). Andes 18: 153-203.

Vitry, C., Soria, S. y G. Pitzzú

2007 La vialidad Inka Calchaquí - Toro, nuevos aportes. Trabajo presentado en el XVI Congreso Nacional de Arqueología Argentina. Disponible en https://www.academia.edu/4088469/La_vialidad_inka_Calchaqu\%C3\%AD_Toro_nu evos_aportes

Wheatley, D.

2004 Making space for an archaeology of place. Internet Archaeology 15:1-16. http: //www. inarch.ac.uk/.

Wheatley, D. y M. Gillings

2000 Vision, perception and GIS: developing enriched approaches to the study of archaeological visibility. Nato Asi Series A: Life Sciences 321: 1-27.

2013 Spatial technology and archaeology: the archaeological applications of GIS. CRC Press, London.

Williams, V. y T. D'Altroy

1998 El sur del Tawantinsuyu: un dominio selectivamente intensivo. Tawantinsuyu 5: 170- 178.

Witcher, R.

1999 GIS and Landscapes of Perception. Geographical Information Systems and Landscape Archaeology, (ed. por M. Gillings, D. Mattingly y J. Van Dalen), pp. 13-22. Oxford.

Wood, J.

1996 The Geomorphological Characterization of Digital Elevation Models. Unpld $\mathrm{PhD}$ Thesis. Leicester.

Zamora Merchán, M.

2006 Visibilidad y SIG en Arqueología: mucho más que ceros y unos. La aplicación de los SIG en la Arqueología del Paisaje, (ed. por Grau Mira, I.), pp. 41-54. Universidad de Alicante, Alicante.

2013 Análisis territorial en arqueología: percepción visual y accesibilidad del entorno. Comechingonia 17(2): 83-106.

Zelarayán, A. y D. Fernández

2015 Linea de base ambiental. Diagnóstico territorial para el ordenamiento del territorio. Ediciones INTA, Salta. 